\title{
Credit card default prediction using machine learning techniques
}

\begin{abstract}
Credit risk plays a major role in the banking industry business. Banks' main activities involve granting loan, credit card, investment, mortgage, and others. Credit card has been one of the most booming financial services by banks over the past years. However, with the growing number of credit card users, banks have been facing an escalating credit card default rate. As such data analytics can provide solutions to tackle the current phenomenon and management credit risks. This paper provides a performance evaluation of credit card default prediction. Thus, logistic regression, rpart decision tree, and random forest are used to test the variable in predicting credit default and random forest proved to have the higher accuracy and area under the curve. This result shows that random forest best describe which factors should be considered with an accuracy of $82 \%$ and an Area under Curve of $77 \%$ when assessing the credit risk of credit card customers.
\end{abstract}

Keyword: Credit score; Data mining; Machine learning; Banking 